\title{
Non-embeddability into a fixed sphere for a family of compact real algebraic hypersurfaces
}

\author{
Xiaojun Huang*, Xiaoshan Li and Ming Xiao
}

\section{Introduction}

Compact CR manifolds of hypersurface type play an important role in the subject of Complex Analysis of Several Variables. For instance, these manifolds include the small link of all isolated complex singularities and, in particular, all exotic spheres of Milnor. In a more geometric aspect, spheres are the model of strongly pseudo-convex hypersurfaces. Motivated by various embedding theorems in differential topology, Stein space theory, etc, it has been a natural question in Several Complex Variables to determine when a real hypersurface $M \subset \mathbb{C}^{n}$ can be holomorphically embedded into the sphere: $\mathbb{S}^{2 N-1}:=\left\{\sum_{j=1}^{N}\left|z_{j}\right|^{2}=1\right\} \subset \mathbb{C}^{N}$ for a sufficiently large $N$.

By a holomorphic embedding of $M \subset \mathbb{C}^{n}$ into $M^{\prime} \subset \mathbb{C}^{N}$, we mean a holomorphic embedding of an open neighborhood $X$ of $M$ into a neighborhood $X^{\prime}$ of $M^{\prime}$, sending $M$ into $M^{\prime}$. It follows easily that a hypersurface holomorphically embeddable into a sphere $\mathbb{S}^{2 N-1}:=\left\{\sum_{j}\left|z_{j}\right|^{2}=\right.$ $1\} \subset \mathrm{C}^{N}$ is necessarily strongly pseudoconvex and real-analytic. However, not every strongly pseudoconvex real-analytic hypersurface can be embedded into a sphere of any dimension, as shown by Forstneric [For] and Faran [Fa] in the mid 1980s based on a Baire category argument. Explicit examples of non-embeddable strongly pseudoconvex real-analytic hypersurfaces were

* Supported in part by NSF-1363418 
given much later by Zaitsev in [Zat] along with explicit invariants serving as obstructions to the embeddability.

A recent observation in [HZ] further shows that if a germ $M$ of a strongly pseudoconvex algebraic hypersurface extends to a germ of algebraic hypersurface with strongly pseudoconcave points or with Levi non-degenerate points of positive signature, then $M$ can not be holomorphically embedded into any sphere.

However, much less is known about the holomorphic embeddability of an open piece of a compact strongly pseudoconvex hypersurface into a sphere. In [HZ], using the local construction in [Zat], the authors gave a compact real analytic strongly pseudoconvex hypersurface, an open piece of which can not embedded into a sphere. Also, in [HZ], it was shown that there are many compact algebraic pseudoconvex hypersurfaces with just one weakly pseudoconvex point any open piece of which can not be embedded into any compact algebraic strongly pseudoconvex hypersurface which, in particular, includes the spheres. For a related work on this, the reader may also consult Ebenfelt and Son [ES]. Here, we should mention a celebrated result of Fornaess [Forn] which states that any compact smooth strongly pseudo-convex hypersurface in a complex Euclidean space can be embedded into a compact strongly convex hypersurface in $\mathbb{C}^{N}$ for a sufficiently large $N$. Though much attention has been paid to the understanding of the embeddability problem as discussed above, the following remains as an open question of long standing:

Open Question: Is any compact strongly pseudoconvex real algebraic hypersurface in $\mathbb{C}^{n}$ $(n \geq 2)$ embeddable into a sphere of a sufficiently large dimension?

Here recall that a smooth real hypersurface in an open subset $U$ of $\mathbb{C}^{n}$ is called real algebraic, if it has a real-valued polynomial defining function.

In this paper, we carry out a study along the lines of the above open question. First, write

$$
M_{\epsilon}=\left\{(z, w) \in \mathbb{C}^{2}: \rho=\varepsilon_{0}\left(|z|^{8}+c \operatorname{Re}|z|^{2} z^{6}\right)+|w|^{2}+|z|^{10}+\epsilon|z|^{2}-1=0\right\} .
$$

Here, $2<c<\frac{16}{7}, \varepsilon_{0}>0$ is a sufficiently small number such that $M_{\varepsilon}$ is smooth for all $0 \leq \epsilon<1$. An easy computation shows that, for any $0<\epsilon<1, M_{\epsilon}$ is strongly pseudoconvex. Also, it is easy to see that $M_{\epsilon}$ is compact. $M_{\epsilon}$ is a small algebraic deformation of the famous KohnNirenberg domain [KN]. Write $D_{\epsilon}$ for the domain bounded by $M_{\epsilon}$. We prove the following 
result in this paper:

Theorem 1.1. For any positive integer $N$, there is a number $\epsilon(N)$ with $0<\epsilon(N)<1$ such that for any $\epsilon$ with $0<\epsilon<\epsilon(N)$, the compact algebraic strongly pseudoconvex hypersurface $M_{\epsilon}$ can not be locally holomorphically embedded into $\mathbb{S}^{2 N-1}$. Namely, for an open piece $U_{\epsilon}$ of $M_{\epsilon}$, any holomorphic map sending $U_{\epsilon}$ into $\mathbb{S}^{2 N-1}$ must be a constant map.

Theorem 1.1 does not give yet a negative answer to the above Open Question. However, it shows at least that the Whitney (or Remmert ) type embedding theorem in differential topology (or in the Stein space theory, respectively) does not hold in the setting considered in this Open Question. We notice that $M_{\epsilon}$ can always be embedded into a generalized sphere with one negative Levi eigenvalue. Indeed, this embedding property is a special case of a general result of Webster [We] which concerns the holomorphic embeddability of an algebraic strongly pseudo-convex hypersurface into a generalized sphere with one negative Levi eigenvalue. Since the Segre families of generalized spheres with the same dimension are biholomorphic to each other, we see that the Segre family of $M_{\epsilon}$ can be holomorphically Segre-embedded into the Segre family of the sphere in $\mathbb{C}^{6}$. We will explain this in more detail in Remark 2.12.

Our proof is based on the algebraicity theorem in [Hu] and the work in Huang-Zaitsev [HZ], where it was shown that $M_{\epsilon}$ can not be embedded into any sphere when $\epsilon=0$. Unfortunately, the compact smooth algebraic hypersurface $M_{\epsilon}$ with $\epsilon=0$ has Kohn-Nirenberg points [KN] which are non-strongly pseudo-convex points. Our family of compact strongly pseudoconvex hypersurfaces are small algebraic perturbation of the Kohn-Nirenberg type domain $M_{0}$. Other main ideas in the paper include the Segre variety technique developed in [HZ] to show the rationality for a certain class of algebraic maps.

\section{Proof of Theorem 1.1}

We divide the proof into many small lemmas for clarity of the exposition.

We first fix needed notations. Let $M \subset U\left(\subset \mathbb{C}^{n}\right)$ be a closed real-analytic subset defined

by a family of real-valued real analytic functions $\left\{\rho_{\alpha}(Z, \bar{Z})\right\}$, where $Z$ is the coordinates of $\mathbb{C}^{n}$. Assume that the complexification $\rho_{\alpha}(Z, W)$ of $\rho_{\alpha}(Z, \bar{Z})$ is holomorphic over $U \times \operatorname{conj}(U)$ 
with

$$
\operatorname{conj}(U):=\{W: \bar{W} \in U\}
$$

for each $\alpha$. Then the complexification $\mathcal{M}$ of $M$ is the complex-analytic subset in $U \times \operatorname{conj}(U)$ defined by $\rho_{\alpha}(Z, W)=0$ for each $\alpha$. Then for $W \in \mathbb{C}^{n}$, the Segre variety of $M$ associated with the point $W$ is defined by $Q_{W}:=\{Z:(Z, \bar{W}) \in \mathcal{M}\}$. In what follows, we will write $\mathcal{M}_{\epsilon}$ for the complexification of $M_{\epsilon}$ and write $\mathcal{M}^{\prime}$ for the complexification of $\partial \mathbb{B}^{N}$. Similarly, we will write $Q_{p}^{\epsilon}$ for the Segre variety of $M_{\epsilon}$ associated with the point $p$, and write $Q_{q}^{\prime}$ for the Segre variety of $\partial \mathbb{B}^{N}$ associated with the point $q$. For any $p \in \mathbb{C}^{2}$, write $p=\left(z_{p}, w_{p}\right)$ or $p=\left(\xi_{p}, \eta_{p}\right)$. The following lemma proved in [HZ] will be used in this paper:

Lemma 2.1. Let $U \subset \mathbb{C}^{n}$ be a simply connected open subset and $\mathcal{S} \subset U$ be a closed complex analytic subset of codimension one. Then for $p \in U \backslash \mathcal{S}$, the fundamental group $\pi_{1}(U \backslash \mathcal{S}, p)$ is generated by loops obtained by concatenating (Jordan) paths $\gamma_{1}, \gamma_{2}, \gamma_{3}$, where $\gamma_{1}$ connects $p$ with a point arbitrary close to a smooth point $q_{0} \in \mathcal{S}, \gamma_{2}$ is a loop around $\mathcal{S}$ near $q_{0}$ and $\gamma_{3}$ is $\gamma_{1}$ reversed.

Making use of the above lemma, we next prove the following lemme: (Notice that a local but a general version of this result played an important role in the paper [HZ].)

Lemma 2.2. Let $M_{\epsilon}$ be defined as in (1.1) with $p_{0}$ in $M_{\epsilon}$. Let $\mathcal{S}$ be a complex analytic hypervariety in $\mathbb{C}^{2}$ not containing $p_{0}$. Let $\gamma \in \pi_{1}\left(\mathbb{C}^{2} \backslash \mathcal{S}, p_{0}\right)$ be obtained by concatenation of $\gamma_{1}, \gamma_{2}, \gamma_{3}$ as described in Lemma 2.1. where $\gamma_{2}$ is a small loop around $\mathcal{S}$ near a smooth point $q_{0} \in \mathcal{S}$ with $w_{q_{0}} \neq 0$. Then $\gamma$ can be slightly and homopotically perturbed to a loop $\widetilde{\gamma} \in \pi_{1}\left(\mathbb{C}^{2} \backslash \mathcal{S}, p_{0}\right)$ such that there exists a null-homotopic loop $\lambda \in \pi_{1}\left(\mathbb{C}^{2} \backslash \mathcal{S}, p_{0}\right)$ with $(\lambda, \overline{\widetilde{\gamma}})$ contained in the complexification $\mathcal{M}_{\epsilon}$ of $M_{\epsilon}$. Also, for an element $\hat{\gamma} \in \pi_{1}\left(\mathbb{C}^{2} \backslash \mathcal{S}, p_{0}\right)$ with a similar property described above, after a small perturbation to $\hat{\gamma}$ if needed, we can find a null-homotopic loop in $\hat{\lambda} \in \pi_{1}\left(\mathbb{C}^{2} \backslash \mathcal{S}, p_{0}\right)$ such that $(\hat{\gamma}, \overline{\hat{\lambda}}) \subset \mathcal{M}_{\epsilon}$.

Proof. First notice the fact that $Q_{p}^{\epsilon}$ is smooth when $w_{p} \neq 0$ defined by $\eta=\varphi(\bar{p}, \xi)$ with $\xi \in \mathbb{C}^{2}$, where $\varphi$ is as in (2.1) below:

$$
\varphi(\bar{p}, \xi)=\varphi\left(\bar{z}_{p}, \bar{w}_{p}, \xi\right)=-\frac{\varepsilon_{0}\left(\xi^{4} \bar{z}_{p}^{4}+\frac{c}{2}\left(\xi^{7} \bar{z}_{p}+\xi \bar{z}_{p}^{7}\right)\right)+\xi^{5} \bar{z}_{p}^{5}+\epsilon \xi \bar{z}_{p}-1}{\bar{w}_{p}},
$$


Moreover, for any $q_{1} \neq q_{2} \in \mathbb{C}^{2}$ with $w_{q_{1}} \neq 0, w_{q_{2}} \neq 0$ and for any $U \subset \mathbb{C}^{2}, Q_{q_{1}}^{\epsilon} \not \equiv Q_{q_{2}}^{\epsilon}$ in $U$ unless they both are empty subset. After slightly perturbing $p_{0}$ in $M_{\epsilon}$, if needed, we can assume without loss of generality that $w_{p_{0}} \neq 0$.

Now for any $\xi \in \mathbb{C}$, we define a map $\mathcal{R}_{\xi}(z, w)=(\xi, \varphi(\bar{z}, \bar{w}, \xi))$ from $\mathbb{C}^{2} \backslash\{w \neq 0\}$ into $\mathbb{C}^{2}$, which is anti-holomorphic in $(z, w)$ for $w \neq 0$ and is real analytic in all variables away from $w=0$. Also, if we write $p_{0}=\left(\xi_{p_{0}}, \eta_{p_{0}}\right)$, then $\left(\xi_{p_{0}}, \varphi\left(\overline{p_{0}}, \xi_{p_{0}}\right)\right)=p_{0}$ and thus $\mathcal{R}_{\xi_{p_{0}}}\left(p_{0}\right)=p_{0}$. From the defintion, we see that $\mathcal{R}_{\xi}$ sends $(z, w)$ to $Q_{(z, w)}^{\epsilon}$.

We claim that, possibly away from a certain nowhere dense closed subset in $\mathbb{C}$ for $\xi$, for a generic smooth point $q$ in the irreducible branch of $\mathcal{S}$ containing $q_{0}$ as in the lemma, there is a sufficiently small ball $\Omega_{q}$ centered at $q$ (whose size may depend on $q$ ) such that $\mathcal{R}_{\xi}$ maps $\Omega_{q}$ into a small open ball $B_{q}$ with $B_{q} \cap \mathcal{S}=\emptyset$. Suppose not. Then we have a smooth piece $E$ from the branch described above of $\mathcal{S}$ such that $\mathcal{R}_{\xi}(E)$ is contained in $\mathcal{S}$ for any $\xi$ in a certain open subset first and then for all $\xi$ by the uniqueness of analytic functions. Letting $\xi=0$, we see that the branch containing all these images must be defined by $z=0$ unless $E$ is defined by $w=$ constant. However, if the branch containing $E$ is defined by $w=$ constant, by making $\xi \neq 0$, we easily see that the union of $\mathcal{R}_{\xi}(E)$ as $\xi$ varies occupies an open subset of $\mathbb{C}^{2}$. This is a contradiction again.

Now, we fix a $\xi_{0}$ as in the above claim and also assume without loss of generality that $\xi_{0}$ is the first coordinate $\xi_{p_{0}}$ of $p_{0}$ (for we are certainly always allowed to perturb $p_{0}$ inside $M_{\epsilon}$ to achieve this). Back to our loop $\gamma$, we now deform $\gamma_{1}, \gamma_{2}, \gamma_{3}$ to $\widetilde{\gamma}_{1}, \widetilde{\gamma}_{2}, \widetilde{\gamma}_{3}$ respectively. Here $\widetilde{\gamma}_{1}$ connects $p_{0}$ with a point $q^{*}$ in a small ball $\Omega$ centered at a certain smooth point $q \in \mathcal{S} \approx q^{*}$, $\widetilde{\gamma}_{2}$ is a loop based at $q^{*}$ around $\mathcal{S}$ inside $\Omega$ and sufficiently close to $q$, and $\widetilde{\gamma}_{3}$ is $\widetilde{\gamma}_{1}$ reserved such that the loop $\widetilde{\gamma}$ obtained by concatenation of $\widetilde{\gamma}_{1}, \widetilde{\gamma}_{2}, \widetilde{\gamma}_{3}$ is the same as $\gamma$ as elements in $\pi_{1}\left(\mathbb{C}^{2} \backslash \mathcal{S}, p_{0}\right)$. Moreover, $\mathcal{R}_{\xi}(\Omega)$ is contained in a ball not cutting $\mathcal{S}$. Also, we assume that the $w$-coordinate of points in $\widetilde{\gamma}(t)$ never vanishes. Now define $\lambda_{2}=\mathcal{R}_{\xi_{0}}\left(\widetilde{\gamma}_{2}\right)$. We choose a suitable path $\{\xi(t): 0 \leq t \leq 1\}$ in $\mathbb{C}$ with $\xi(0)=\xi(1)=\xi_{0}$ such that if we define $\lambda_{1}=\mathcal{R}_{\xi(t)}\left(\widetilde{\gamma}_{1}\right)$, then $\lambda_{1}$ avoids $\mathcal{S}$ (with possibly a slight perturbation of $\widetilde{\gamma}_{1}$ fixing endpoints). Furthermore, if we define $\lambda_{3}$ to be the reverse of $\lambda_{1}$, and $\lambda$ to be the concatenation of $\lambda_{1}, \lambda_{2}, \lambda_{3}$, then $\lambda$ is a nullhomotopic loop in $\pi_{1}\left(\mathbb{C}^{2} \backslash \mathcal{S}, p_{0}\right)$. Moreover, $(\lambda(t), \bar{\gamma}(t))$ is in the complexification $\mathcal{M}_{\epsilon}$ of $M_{\epsilon}$ by the way it was constructed. The last statement in the lemma follows from the symmetric property of Segre variety and what we just proved. 
Proposition 2.3. For an $\epsilon$ with $0<\epsilon<1$, assume that $F$ is non-constant holomorphic map from an open piece of $M_{\epsilon}$ into $\partial \mathbb{B}^{N}(N \in \mathbb{N})$. Then $F$ extends to a proper rational map from $D_{\epsilon}$ into $\mathbb{B}^{N}$, holomorphic over $\overline{D_{\epsilon}}$.

Proof. By a theorem of the first author in $[\mathrm{Hu}], F$ is complex algebraic (possibly multi-valued). In particular, any branch of $F$ can be holomorphically continued along a path not cutting a certain proper complex algebraic subset $\mathcal{S} \subset \mathbb{C}^{2}$. We need only to prove the proposition assuming that $\mathcal{S}$ is a hyper-complex analytic variety. Seeking a contradiction, suppose not. Then we can find a point $p_{0} \in U \subset M_{\epsilon}, p_{0}=\left(z_{0}, w_{0}\right)$ with $w_{0} \neq 0$, a loop $\gamma \in \pi_{1}\left(\mathbb{C}^{2} \backslash \mathcal{S}, p_{0}\right)$ obtained by concatenation of $\gamma_{1}, \gamma_{2}, \gamma_{3}$ as in Lemma 2.1, where $\gamma_{2}$ is a small loop around $\mathcal{S}$ near a smooth point $q_{0} \in \mathcal{S}$, such that when we holomorphically continue $F$ from a neighborhood of $p_{0}$ along $\gamma$ one round, we will obtain another branch $F_{2}(\neq F)$ of $F$ near $p_{0}$. Obviously, we can assume $q_{0}$ is a smooth point of some branching hypervariety $\mathcal{S}^{\prime} \subset \mathcal{S}$ of $F$. We next proceed in two steps:

Case I: If we can find a loop $\gamma$ as above such that the corresponding $\mathcal{S}^{\prime} \neq\{w=0\}$, by perturbing $\gamma$ if necessary, we can make $w_{q_{0}} \neq 0$. By Lemma 2.2. after slightly perturbing $\gamma$ if necessary, there exists a null-homotopic loop $\lambda$ in $\pi_{1}\left(\mathbb{C}^{2} \backslash \mathcal{S}, p_{0}\right)$ with $(\gamma, \bar{\lambda})$ contained in the complexification $\mathcal{M}_{\epsilon}$ of $M_{\epsilon}$ We know that $(F, \bar{F}):=(F(\cdot), \overline{F(\cdot)})$ sends a neighborhood of $\left(p_{0}, \overline{p_{0}}\right)$ in $\mathcal{M}_{\epsilon}$ into $\mathcal{M}^{\prime}$. Applying the analytic continuation along the loop $(\gamma, \bar{\lambda})$ in $\mathcal{M}_{\epsilon}$ for $\rho(F, \bar{F})$, one concludes by the uniqueness of analytic functions that $\left(F_{2}, \bar{F}\right)$ also sends a neighborhood of $\left(p_{0}, \overline{p_{0}}\right)$ in $\mathcal{M}_{\epsilon}$ into $\mathcal{M}^{\prime}$. Consequently, we get $F_{2}\left(Q_{p}\right) \subset Q_{F(p)}^{\prime}$ for $p \in M_{\epsilon}$ near $p_{0}$. In particular, we have the following:

$$
F_{2}(p) \in Q_{F(p)}^{\prime}, \forall p \in M_{\epsilon}, p \approx p_{0} .
$$

Now applying the holomorphic continuation along the loop $(\lambda, \bar{\gamma})$ in $\mathcal{M}_{\epsilon}$ for $\rho\left(F_{2}, F\right)$, we get by uniqueness of analytic functions that $\left(F_{2}, \overline{F_{2}}\right)$ sends a neighborhood of $\left(p_{0}, \overline{p_{0}}\right)$ in $\mathcal{M}_{\epsilon}$ into $\mathcal{M}^{\prime}$. Hence, we also have

$$
F_{2}(p) \in Q_{F_{2}(p)}^{\prime}, \forall p \in M_{\epsilon}, p \approx p_{0} .
$$

In particular, $F_{2}(p) \in \partial \mathbb{B}^{N}$. Combining this with equation (2.2), and noting that for any $q \in \partial \mathbb{B}^{N}, \partial \mathbb{B}^{N} \cap Q_{q}^{\prime}=q$, we get $F_{2}(p)=F(p)$ for any $p \in M_{\epsilon}$ near $p_{0}$. Thus $F_{2} \equiv F$ in a neighborhood of $p_{0}$ in $\mathbb{C}^{2}$, which is a contradiction. 
Case II: Now, suppose $W:=\{w=0\}$ is the only branching locus of the algebraic extension of $F$. Since $W$ is smooth and $\pi_{1}\left(\mathbb{C}^{2} \backslash W\right)=\mathbb{Z}$, we get the cyclic branching property for $F$. Now, we notice that $W$ cuts $M_{\epsilon}$ transversally at a certain point $p^{*}=:\left(z_{0}, 0\right)$. When we will continue along loops inside $T_{p^{*}}^{(1,0)} M_{\epsilon}$ near $p^{*}$, we recover all branches of $F(z, w)$. Since any loop inside $T_{p^{*}}^{(1,0)} M_{\epsilon}$ near $p^{*}$ can be easily homotopically deformed into loops in $M_{\epsilon}$ near $p^{*}$, we conclude that we recover all branches of $F$ near $p^{*}$ by continuing any branch of $F$ near $p^{*}$ along loops inside $M_{\epsilon} \backslash W$ near $p^{*}$. Hence, we are now reduced to the local situation as encountered in Proposition 3.10 of [HZ]. Hence, by Proposition 3.10 of [HZ], for $Z(\neq) \approx p^{*}$ and two barnches $F_{1}$ and $F_{2}$ of $F$ near $Z$, we have $F_{1}(Z), F_{2}(Z) \in Q_{F_{1}(Z)}^{\prime} \cap Q_{F_{2}(Z)}^{\prime}$. As above, we see that $F_{1}(Z)=F_{2}(Z)$. We thus conclude that $F$ is single-valued.

Since $F$ is algebraic, it is rational. Once we know that $F$ is a rational map from $M_{\epsilon}$ into the sphere, by a theorem of Chiappari [Ch], we know that $F$ extends to a holomorphic map from a neighborhood of $\overline{D_{\epsilon}}$ and properly maps $D_{\epsilon}$ into the ball. This completes the proof of Proposition 2.3.

Next we recall the following definition.

Definition 2.4. Let $F$ be a rational map from $\mathbb{C}^{n}$ into $\mathbb{C}^{m}$. We write

$$
F=\frac{\left(P_{1}, \cdots, P_{m}\right)}{R}
$$

where $P_{j}, j=1, \cdots, m$ and $R$ are holomorphic polynomials and $\left(P_{1}, \cdots, P_{m}, R\right)=1$. The degree of $F$, denoted by $\operatorname{deg} F$, is defined to be

$$
\operatorname{deg} F:=\max \left\{\operatorname{deg}\left(P_{j}\right), j=1, \cdots, m, \operatorname{deg} R\right\} .
$$

To emphasize on the dependence on the parameter $\epsilon$, in what follows, we write $F^{\epsilon}$ for a holomorphic map from a certain open piece of $M_{\epsilon}$ into $\partial \mathbb{B}^{N}$. By what we did above, $F^{\epsilon}$ extends to holomorphic map over a neighborhood of $\overline{D_{\epsilon}}$. The purpose of the next three lemmas is to show the uniform boundedness of the degree of $F^{\epsilon}$. We mention a related article of Meylan in [Mey] for the uniform estimate of degree for proper rational maps between balls. 
Lemma 2.5. Let $F^{\epsilon}$ be a proper rational map from $D_{\epsilon}$ into $\mathbb{B}^{N}$ holomorphic over $\overline{D_{\epsilon}}$. Then there is an open piece $U$ of $M_{\epsilon}$ such that for any $p \in U$ with $w_{p} \neq 0$ and we have $\left.\operatorname{deg} F^{\epsilon}\right|_{Q_{p}} \leq d$, where $d=\frac{7 N(N+1)}{2}$. Here we set $\left.F^{\epsilon}\right|_{Q_{p}}:=F^{\epsilon}\left(\xi, \phi\left(\overline{z_{p}}, \overline{w_{p}}, \xi\right)\right)$ with $\phi\left(\overline{z_{p}}, \overline{w_{p}}, \xi\right)$ as in (2.1), which is a holomorphic polynomial function in $\xi$.

Proof. Let $p_{0}=\left(z_{0}, w_{0}\right) \in M_{\epsilon}$ with $w_{p_{0}} \neq 0$. For any $(\xi, \eta) \in Q_{p_{0}}$, we have

$$
F_{1}^{\epsilon}(z, w) \overline{F_{1}^{\epsilon}(\xi, \eta)}+\cdots+F_{N}^{\epsilon}(z, w) \overline{F_{N}^{\epsilon}(\xi, \eta)}=1,(z, w) \in Q_{(\xi, \eta)} .
$$

Here we write $F^{\epsilon}=\left(F_{1}^{\epsilon}, \cdots, F_{N}^{\epsilon}\right)$. Recall $Q_{(\xi, \eta)}$ is given by $\varepsilon_{0}\left(z^{4} \bar{\xi}^{4}+\frac{c}{2}\left(\bar{\xi} z^{7}+z \bar{\xi}^{7}\right)\right)+w \bar{\eta}+$ $z^{5} \bar{\xi}^{5}+\epsilon z \bar{\xi}-1=0$. Write

$$
\mathcal{L}=\left(4 \varepsilon_{0} \bar{\xi}^{4} z^{3}+\frac{7 c \varepsilon_{0}}{2} \bar{\xi} z^{6}+\frac{c \varepsilon_{0}}{2} \bar{\xi}^{7}+5 \bar{\xi}^{5} z^{4}+\epsilon \bar{\xi}\right) \frac{\partial}{\partial w}-\bar{\eta} \frac{\partial}{\partial z}
$$

Then $\mathcal{L}$ forms a basis for the holomorphic tangent vector fields of $Q_{(\xi, \eta)}$ near $(z, w) \in Q_{(\xi, \eta)}$. When $(\xi, \eta)=(z, w)$ and moves along $U \subset M_{\epsilon}, \mathcal{L}$ reduces to the $\mathrm{CR}$ vector field along $U \subset M_{\epsilon}$. Applying $\mathcal{L}^{\alpha},|\alpha|>0$, to (2.4) and evaluating at $p_{0}$, one gets

$$
\mathcal{L}^{\alpha} F_{1}^{\epsilon}\left(z_{0}, w_{0}\right) \overline{F_{1}^{\epsilon}(\xi, \eta)}+\cdots+\mathcal{L}^{\alpha} F_{N}^{\epsilon}\left(z_{0}, w_{0}\right) \overline{F_{N}^{\epsilon}(\xi, \eta)}=0,|\alpha|>0
$$

Write

$$
V_{\alpha}^{\epsilon}(\xi, \eta)=\left(\mathcal{L}^{\alpha} F_{1}^{\epsilon}\left(z_{0}, w_{0}\right), \cdots, \mathcal{L}^{\alpha} F_{N}^{\epsilon}\left(z_{0}, w_{0}\right)\right) .
$$

Choose $U \subset M_{\epsilon}$ such that $\left\{V_{\alpha}^{\epsilon}\left(z_{0}, w_{0}\right)\right\}_{\alpha>0}^{\infty}$ has a constant rank $k \leq N$ for $\left(z_{0}, w_{0}\right) \in U$. Then, after shrinking $U$ if needed, by a calculus computation (see [La], for instance) we conclude that $\left\{V_{\alpha}^{\epsilon}\left(z_{0}, w_{0}\right)\right\}_{\alpha>0}^{k}$ must be a basis of $\left\{V_{\alpha}^{\epsilon}\left(z_{0}, w_{0}\right)\right\}_{\alpha>0}^{\infty}$. Making use of the Taylor expansion, we see that the linear span of $\left\{V_{\alpha}^{\epsilon}\left(z_{0}, w_{0}\right)\right\}_{\alpha>0}^{k}$ is the smallest subspace containing $F^{\epsilon}\left(Q_{\left(z_{0}, w_{0}\right)}\right)-F^{\epsilon}\left(z_{0}, w_{0}\right)$.

- If $k=N-1$ in $U$, we can solve for $F^{\epsilon}(\xi, \eta)$ for $(\xi, \eta) \in Q_{\left(z_{0}, w_{0}\right)}$ from Equation (2.4) and (2.6) by the Cramer rule. Notice that $\eta=\phi\left(\overline{p_{0}}, \xi\right)$ is solved as a polynomial function of $\xi$ of degree 7 . Therefore, as a rational function in $\xi$, we get

$$
\left.\operatorname{deg} F^{\epsilon}\right|_{Q_{\left(z_{0}, w_{0}\right)}} \leq d
$$

for $\left(z_{0}, w_{0}\right) \in U$. 
- If $k<N-1$, then one can find constant vectors $\mathbf{V}_{1}, \cdots, \mathbf{V}_{N-k}$ in $\mathbb{C}^{N}$ such that

$$
\operatorname{Span}\left\{\mathbf{V}_{1}, \cdots, \mathbf{V}_{N-k}\right\} \bigoplus \operatorname{Span}\left\{V_{\alpha}^{\epsilon}\left(z_{0}, w_{0}\right)\right\}_{1 \leq \alpha \leq k}=\mathbb{C}^{N-1}
$$

and $\mathbf{V}_{i} \cdot\left(\overline{F^{\epsilon}(\xi, \eta)-F^{\epsilon}\left(z_{0}, w_{0}\right)}\right)=0$ on $Q_{\left(z_{0}, w_{0}\right)}, 1 \leq i \leq N-k$. One can still apply Cramer's rule to solve for $F^{\epsilon}(\xi, \eta)$ with $(\xi, \eta) \in Q_{\left(z_{0}, w_{0}\right)}$ to show, as a rational function of $\xi$, that

$$
\left.\operatorname{deg} F^{\epsilon}\right|_{Q_{\left(z_{0}, w_{0}\right)}}<d
$$

This completes the proof of the lemma.

Remark: The above argument can be use to show directly that $F$ is rational (as a function in $\xi$ ) when restricted to a Segre variety. However this type of information is not enough, in general, to conclude the rationality of $F$ : Let $M \subset \mathbb{C}^{2}$ be a strongly pseudoconvex hypersurface defined by $|w|^{2}=\left(1+|z|^{2}\right)^{2}$ and $g=\sqrt{w}$. The Segre variety $Q_{(z, w)}$ of $M$ for each $(z, w)$ is defined by $w \bar{\eta}=\left.(1+z \bar{\xi})^{2} \cdot g\right|_{Q_{(z, w)}}= \pm \frac{1+\bar{z} \xi}{\sqrt{w}}$, which is a polynomial as a function in $\xi$ for $w \neq 0$.

Lemma 2.6. Let $H=\frac{\left(P_{1}, \cdots, P_{N}\right)}{R}$ with $R(0,0) \neq 0$ be a rational map from $\mathbb{C}^{2} \backslash\{R=0\}$ into $\mathbb{C}^{N}$, where $P_{j}, j=1, \cdots, N, R$ are holomorphic polynomials and $\left(P_{1}, \cdots, P_{N}, R\right)=1$. Assume that there is an open subset $U$ of $M_{\epsilon}$ such that for each $p \in U$ with $w_{p} \neq 0$ and, as a rational function in $\xi \operatorname{deg}\left(\left.H\right|_{Q_{p}}\right) \leq k$ with $k>0$ a fixed integer. Then $\operatorname{deg}(H) \leq k$.

Proof. Set

$$
A=\left\{(\xi, \eta) \in \mathbb{C}^{2}: P_{1}(\xi, \eta)=\cdots=P_{N}(\xi, \eta)=R(\xi, \eta)=0\right\} .
$$

Then $A$ has at most finitely many points. It is easy to see that if $Q_{p}$ does not pass through any point of $A$, then as a rational function in $\xi$, the degree of $\left.H\right|_{Q_{p}}$ is the same as the degree of $H$ as a rational function in all variables. Thus it only remains to show the existence of $\left(z_{0}, w_{0}\right) \in U$ such that $Q_{\left(z_{0}, w_{0}\right)} \cap A=\emptyset$. Indeed, fix $\left(\xi_{0}, \eta_{0}\right) \in A$, then $\xi_{0} \neq 0$ or $\eta_{0} \neq 0$. $\left(\xi_{0}, \eta_{0}\right) \in Q_{\left(z_{0}, w_{0}\right)}$ if and only if

$$
\varepsilon_{0} \bar{\xi}_{0}^{4} z_{0}^{4}+\frac{c}{2} \varepsilon_{0}\left(\bar{\xi}_{0} z_{0}^{7}+z \bar{\xi}_{0}^{7}\right)+w_{0} \bar{\eta}_{0}+z_{0}^{5} \bar{\xi}_{0}^{5}+\epsilon z_{0} \bar{\xi}_{0}=1
$$

The collection of such pairs $\left\{\left(z_{0}, w_{0}\right)\right\}$ is a complex subvariety of complex dimension 1 . Thus $\left\{(z, w) \in \mathbb{C}^{2}: Q_{(z, w)} \cap A \neq \emptyset\right\}$ is a finite union of complex subvarieties of complex dimension 1 . But $U \subset M_{\epsilon}$ is of real dimension 3. Thus there exists $\left(z_{0}, w_{0}\right) \in U$ such that $Q_{\left(z_{0}, w_{0}\right)} \cap A=\emptyset$. 
Notice that our $F^{\epsilon}$ is holomorphic in $D_{\epsilon}$ and thus at 0 . As a consequence of Lemma 2.5 and Lemma 2.6, we have the following:

Lemma 2.7. Let $F^{\epsilon}, d$ be as in Lemma 2.5. Then $\operatorname{deg} F^{\epsilon} \leq d$.

The following three lemmas are to show the uniform boundedness of the coefficients of $F^{\epsilon}$.

Lemma 2.8. Let $p(z)=\sum_{i=1}^{m} a_{i} z^{i}+1$ be a holomorphic polynomial in $\mathbb{C}$. Assume that $p(z) \neq 0$ in $\Delta$, where $\Delta$ is the unit disk centered at 0 in $\mathbb{C}$. Then $\left|a_{i}\right| \leq C_{m}$ for all $1 \leq i \leq m$, where $C_{m}$ is a constant depending only on $m$. Consequently, $|p(z)| \leq m C_{m}+1$ in $\Delta$.

Proof. We write $p(z)=a_{k} \Pi_{i=1}^{k}\left(z-z_{i}\right)$, where $1 \leq k \leq m$ is the largest number $l$ such that $a_{l} \neq 0$, and $\left\{z_{i}\right\}_{i=1}^{k}$ are the roots of $p(z)$ in $\mathbb{C}$. Notice that $p(0)=1$ and $p(z) \neq 0$ in $\Delta$, we get $\left|z_{i}\right| \geq 1$ for all $1 \leq i \leq k$, and $\left|a_{k} \Pi_{i=1}^{k} z_{i}\right|=1$. Thus $\left|a_{k}\right| \leq 1$. Moreover, by applying Vieta's formula, we have for each $1 \leq j \leq k-1$,

$$
\left|a_{k-j}\right|=\left|\frac{\sum_{l_{1}<\cdots<l_{j}} z_{l_{1}} \cdots z_{l_{j}}}{\Pi_{i=1}^{k} z_{i}}\right| \leq C_{m}
$$

for a certain constant $C_{m}$ depending only on $m$.

Lemma 2.9. Let $p(z)=\sum_{|\alpha|=1}^{m} a_{\alpha} z^{\alpha}+1$ be a holomorphic polynomial in $\mathbb{C}^{N}, N \geq 1$. Assume that $p(z) \neq 0$ in $\mathbb{B}^{N}$. Then $\left|a_{\alpha}\right| \leq \widetilde{C}_{m}$ for all $1 \leq|\alpha| \leq m$, where $\widetilde{C}_{m}$ is a positive constant depending only on $m$.

Proof. Fix $z \in \partial \mathbb{B}^{N}$. Set $\tilde{p}(\xi)=p(\xi z), \xi \in \Delta$, which is a holomorphic polynomial in $\mathbb{C}$. Noting that $\tilde{p}(\xi) \neq 0$ in $\Delta$, by Lemma 2.8, $|\tilde{p}(\xi)| \leq m C_{m}+1$, where $C_{m}$ is as in Lemma 2.8. Consequently, $|p(z)| \leq m C_{m}+1, \forall z \in \mathbb{B}^{N}$. By the Cauchy estimate, we conclude that there exists some constant $\widetilde{C}_{m}$ such that $\left|a_{\alpha}\right| \leq \widetilde{C}_{m}$ for all $1 \leq|\alpha| \leq m$.

Lemma 2.10. Let $F^{\epsilon}, d$ be as in Lemma 2.5 and assume that $F^{\epsilon}(0)=0$. Write $F^{\epsilon}(z, w)=$ $\frac{P^{\epsilon}(z, w)}{Q^{\epsilon}(z, w)}$, where $P^{\epsilon}(z, w)=\sum_{1 \leq i+j \leq d} a_{i j}^{\epsilon} z^{i} w^{j}, Q^{\epsilon}(z, w)=\sum_{1 \leq i+j \leq d} b_{i j}^{\epsilon} z^{i} w^{j}+1$. Moreover $\left(P^{\epsilon}, Q^{\epsilon}\right)=1$. Then $\left|a_{i j}^{\epsilon}\right| \leq C,\left|b_{i j}^{\epsilon}\right| \leq C$ for some constant $C$ depending only on $N$.

Proof. Notice that there exists $r>0$ independent of $0<\epsilon<1$ such that $B(0, r) \subset D_{\epsilon}$ and $Q^{\epsilon}(z, w) \neq 0$ in $B(0, r)$. As an application of Lemma 2.9, one can show the uniform 
boundedness of $\left|b_{i j}^{\epsilon}\right|$ by considering $\tilde{Q}^{\epsilon}(z, w)=Q^{\epsilon}(\sqrt{r} z, \sqrt{r} w)$. Consequently, $P^{\epsilon}$ is uniformly bounded in $B(0, r)$ for all $\epsilon$. And the uniform boundedness of $a_{i j}^{\epsilon}$ follows from the Cauchy estimate.

Set $M_{0}=\left\{(z, w) \in \mathbb{C}^{2}: \rho=\varepsilon_{0}\left(|z|^{8}+c \operatorname{Re}|z|^{2} z^{6}\right)+|w|^{2}+|z|^{10}-1=0\right\}$. Notice that $M_{0}$ has the Kohn-Nirenberg property at the point $(0,1)$. Here recall that (see [HZ]) a real hypersurface $M \subset \mathbb{C}^{n}$ is said to satisfy the Kohn-Nirenberg property at $p \in M$, if for any holomorphic function $h \not \equiv 0$ in any neighborhood $U$ of $p$ in $\mathbb{C}^{n}$ with $h(p)=0$, the zero set $\mathcal{Z}$ of $h$ intersects $M$ transversally at some smooth point of $\mathcal{Z}$ near $p$. As an immediate application of Theorem 3.6 in [HZ], one has the following lemma,

Lemma 2.11. Let $M_{0}$ be as above. Then any holomorphic map sending an open piece of $M_{0}$ into $\partial \mathbb{B}^{N}$ is a constant.

We are now ready to prove our main theorem.

Proof of Theorem 1.1. Seeking a contradiction, suppose the statement in the main theorem does not hold. Then for a certain positive integer $N$ and for a certain sequence $1>\epsilon_{k} \rightarrow 0^{+}, M_{\epsilon_{k}}$ are locally holomorphically embeddable into $\mathbb{S}^{2 N-1}$ for any $\epsilon_{k}$. For each of such $\epsilon_{k}$, write a local holomorphic embedding as $F^{\epsilon_{k}}$. Then, by Lemma 2.3 , $F^{\epsilon_{k}}$ extends to a rational and holomorphic map over $\overline{D_{\epsilon}}$. After composing with an automorphism of $\mathbb{B}^{N}$, we can assume that $F^{\epsilon_{k}}(0)=0$.

By Lemma 2.7 and Lemma 2.10, we can write

$$
F^{\epsilon_{k}}(z, w)=\frac{\sum_{i+j=1}^{d} a_{i j}^{\epsilon_{k}} z^{i} w^{j}}{\sum_{i+j=1}^{d} b_{i j}^{\epsilon_{k}} z^{i} w^{j}+1}
$$

where $d=\frac{7 N(N+1)}{2}$ and $\left|a_{i j}^{\epsilon_{k}}\right| \leq C,\left|b_{i j}^{\epsilon_{k}}\right| \leq C$ for all $i, j$ with $C$ a constant as in Lemma 2.10, Hence after passing to a subsequence if necessary, we can assume that $a_{i j}^{\epsilon_{k}} \rightarrow a_{i j}, b_{i j}^{\epsilon_{k}} \rightarrow b_{i j}$ as $k \rightarrow \infty$ for some $a_{i j} \in \mathbb{C}, b_{i j} \in \mathbb{C}$ for all $i, j$. Set $F(z, w)=\frac{P(z, w)}{Q(z, w)}$, where $P(z, w)=\sum_{i+j=1}^{d} a_{i j} z^{i} w^{j}$ and $Q(z, w)=\sum_{i+j=1}^{d} b_{i j} z^{i} w^{j}+1$. Let $V=\left\{(z, w) \in \mathbb{C}^{2}: Q(z, w)=0\right\}$ be the variety defined 
by the zeros of $Q(z, w)$ in $\mathbb{C}^{2}$. It is easy to see that for any open subset $K \subset \subset \mathbb{C}^{2} \backslash V$, we have $F^{\epsilon_{k}}$ converges to $F$ uniformly in $K$. Pick $p_{0} \in M \backslash V$ and a neighborhood $U$ of $p_{0}$ with $U \subset \subset \mathbb{C}^{2} \backslash V . F^{\epsilon_{k}}$ converges to $F$ uniformly in $\bar{U}$. Notice that for any $p \in U \cap M$, there exists $p_{k} \in M_{\epsilon_{k}}$ such that $p_{k} \rightarrow p$ as $k \rightarrow \infty$. Then $\|F(p)\|=\lim _{k \rightarrow \infty}\left\|F^{\epsilon_{k}}\left(p_{k}\right)\right\|=1$. By Lemma 2.11, $F$ is a constant map from $D_{\epsilon} \cap M$ into the sphere. This is a contradiction, for we know that $F(0)=0$. The proof of Theorem 1.1 is complete.

Remark 2.12. It is clear that with the same proof, we can construct a lot of more similar examples as in Theorem 1.1 .

Next, to see that $M_{\epsilon}$ can be holomorphically embedded into the generalized sphere in $\mathbb{C}^{6}$ with one negative Levi eigenvalue, we observe that $\operatorname{Re}\left(|z|^{2} z^{6}\right)=\frac{1}{4}\left(\left|z^{7}+z\right|^{2}-\left|z^{7}-z\right|^{2}\right)$. Thus the map

$$
F(z, w)=\left(\sqrt{\varepsilon_{0}} z^{4}, \frac{1}{2} \sqrt{\varepsilon_{0} c}\left(z^{7}+z\right), w, z^{5}, \sqrt{\epsilon} z, \frac{1}{2} \sqrt{\varepsilon_{0} c}\left(z^{7}-z\right)\right)
$$

holomorphically embeds $M_{\epsilon}$ into the generalized sphere in $\mathbb{C}^{6}$ defined by $\mathbb{S}^{11}=\left\{\left(Z_{1}, \cdots, Z_{6}\right) \in\right.$ $\left.\mathbb{C}^{6}: \sum_{j=1}^{5}\left|Z_{j}\right|^{2}-\left|Z_{6}\right|^{2}=1\right\}$.

\section{References}

[Ch] S. Chiappari, Holomorphic extension of proper meromorphic mappings, Mich. Math. J. 38, 167- 174 (1991).

[ES] P. Ebenfelt and D. Son, On the existence of holomorphic embeddings of strictly pseudoconvex algebraic hypersurfaces into spheres, May, 2012. (arXiv:1205.1237).

[Fa] J. J. V. Faran, The nonimbeddability of real hypersurface in spheres. Proc. Amer. Math. Soc.103, 3(1988), 902-904.

[Forn] J. E. Fornæss, Strictly pseudoconvex domains in convex domains, Amer. J. Math. 98 (1976), 529-569.

[For] F. Forstneric, Embedding strictly pseudoconvex domains into balls, Transations of AMS (Vol.295), No.1 (May, 1986), 347-368. 
[HJ] X. Huang and S. Ji, Mapping $\mathbb{B}^{n}$ into $\mathbb{B}^{2 n-1}$, Invent.Math., 145(2001), 219-250.

[Hu] X.Huang, On the mapping problem for algebraic real hypersurfaces in the complex spaces of different dimensions, Annales de l'institut Fourier, 44.2(1994) 433-463.

[HZ] X. Huang, D. Zaitsev, Non-embeddable real algebraic hypersurface, Math. Z., 275, No. 3-4, 657-671 (2013).

[KN] J. J. Kohn and L. Nirenberg, A pseudo-convex domain not admitting a holomorphic support function, Math. Ann., 201 (1973), 265-268.

[La] B. Lamel, A reflection principle for real-analytic submanifolds of complex spaces, J. Geom. Anal. 11 , no. 4, 625-631, (2001).

[Mey] F. Meylan, Degree of a holomorphic map between unit balls from $\mathbb{C}^{2}$ to $\mathbb{C}^{n}$, Proc. Amer. Math. Soc. 134 (2006), 1023-1030.

[We] S. M. Webster, Some birational invariants for algebraic real hypersurfaces, Duke Math. J., Volume 45, Number 1 (1978), 39-46.

[Zat] D. Zaitsev, Obstructions to embeddability into hyperquadrics and explicit examples, Math. Ann., 342 (2008), no. 3, 695-726.

X. Huang, Department of Mathematics, Rutgers University at New Brunswick, New Jersey 08903, USA. (huangx@math.rutgers.edu)

X. Li, School of Mathematics and Statistics, Wuhan University, Hubei, Wuhan 430072, China. (xiaoshanli@whu.edu.cn)

M. Xiao, Department of Mathematics, Rutgers University at New Brunswick, New Jersey 08903, USA. (mingxiao@math.rutgers.edu) 